ISSN: 2591-7994

\title{
Implementation of a restrictive blood transfusion protocol in a gynecologic oncology service.
}

\author{
Jaron Mark ${ }^{1 *}$, Sarah Lynam¹, Kayla Morrell ${ }^{2}$, Kevin Eng ${ }^{2}$, Kristen Starbuck ${ }^{1}$, J Brian Szender', Emese Zsiros ${ }^{1}$, \\ Peter J Frederick ${ }^{1}$ \\ ${ }^{1}$ Department of Gynecologic Oncology, Roswell Park Comprehensive Cancer Center, Buffalo, New York, USA \\ ${ }^{2}$ Department of Biostatistics, Roswell Park Comprehensive Cancer Center, Buffalo, New York, USA
}

\begin{abstract}
Objectives: The purpose of this study was to evaluate the impact of a restrictive blood transfusion protocol in a postoperative gynecologic oncology population. The primary objective was the rate of blood transfusions after surgery before and after implementation of a restrictive transfusion protocol (from July $1^{\text {st }} 2011$ to December $30^{\text {th }}$ 2016). Secondary outcomes were patient morbidity and included rates of surgical site infection, pneumonia, sepsis, unplanned intubation, prolonged ventilator use, renal insufficiency, acute renal failure, urinary tract infection, cerebral vascular accident, cardiac complications, venous thromboembolism, and death within 30 days of surgery, readmissions and length of stay.
\end{abstract}

Methods: A restrictive blood transfusion protocol was implemented by the gynecologic oncology service at a National Comprehensive Cancer Network designated Comprehensive Cancer Center on January $1^{\text {st }}, 2014$. The restrictive protocol required that no patient receive a blood transfusion for hemoglobin greater than $7.0 \mathrm{~g} / \mathrm{dL}$ (or hematocrit greater than $\mathbf{2 1 . 0} \%$ ) and that all red blood cells were administered in one unit increments followed by re-evaluation of blood parameters. Exceptions to this protocol were postoperative symptomatic anemia, intraoperative or day of surgery transfusion, active bleeding, postoperative severe sepsis, postoperative active coronary ischemia, and postoperative transfusion after 1.5 liter or greater blood loss.

Results: 1482 patients were identified for this study (755 in the pre-protocol group and 727 in the post-protocol group). Patients treated under the restrictive protocol had decreased rates of red blood cell transfusion (11.0\% vs $5.9 \%$ p $<0.001)$, superficial surgical site infection $(7.7 \%$ vs $4.1 \%$ $\mathrm{p}=\mathbf{0 . 0 0 5})$, deep surgical site infection $(2.3 \%$ vs $0.7 \% \mathrm{p}=0.02)$, and median length of stay (3.0 days vs 2.0 days $\mathrm{p}<\mathbf{0 . 0 0 1 )}$.

Conclusions: A restrictive blood transfusion protocol is associated with reductions in the rates of blood transfusions and postoperative morbidity with a $46.8 \%$ reduction in superficial surgical site infection and a $\mathbf{6 9 . 6 \%}$ decrease in deep surgical site infection in the gynecologic oncology patient population.

Keywords: Blood transfusion, Restrictive transfusion, Gynecology oncology, Gynecologyy

Accepted on January 04, 2019

\section{Introduction}

According to the American Red Cross, approximately 36,000 units of red blood cells are needed daily in the United States with an estimated 21 million blood components transfused each year [1]. Annual cost estimates range from $\$ 2.5$ to $\$ 12.5$ billion when accounting for hospital-associated costs with blood product administration [2]. Frequent blood component shortages in the United States further stress the importance of mitigating unnecessary transfusions [3]. Changes in transfusion practices on any scale have the potential to reduce healthcare costs by billions of dollars per year. Blood transfusion guidelines, if they even exist, are inconsistently applied and vary widely from nation to nation, region to region, hospital to hospital and individual surgeon to surgeon as do various medical societal guidelines $[4,5]$. Often, transfusion practices are guided by dogma rather than evidence-based medicine.

Red blood cell transfusion has been historically used to improve hemoglobin values to $10 \mathrm{~g} / \mathrm{dL}$ or greater [6]. Mounting evidence suggests transfusions are not a benign intervention and are associated with increased rates of infection, hemolytic reactions, anaphylaxis, transfusion-associated circulatory overload, sepsis, transfusion-related acute lung injury and death [7]. An estimated 50,000 transfusion reactions occur in the United States annually [2]. Most fatalities are a result of transfusion-associated sepsis, hemolytic transfusion reactions and transfusion-related acute lung injury [8]. Level II evidence has shown that blood transfusion protocols that are not restrictive are associated with increased patient morbidity and mortality [9]. A 2016 Cochrane review of 12,587 patients analyzed the safety of transfusing to maintain hemoglobin of 7-8 g/dL vs 9-10 g/dL [10]. The included studies encompassed orthopedic surgery, critical care, trauma, cardiac surgery, acute coronary syndrome, leukemia, hematological cancer, vascular surgery and pediatric patients. Analyses included 30 day mortality, cardiac events (myocardial infarction and heart failure), stroke, thromboembolism, sepsis, pneumonia, postoperative wound infection, renal failure, mental 
confusion and functional recovery. No statistical differences in these clinical variables were observed between the restrictive and liberal transfusion groups. These results suggest that a restrictive transfusion protocol which transfused patients at a hemoglobin concentration of $7 \mathrm{~g} / \mathrm{dL}$ to $8 \mathrm{~g} / \mathrm{dL}$ was not harmful and that the potential negative effects of blood transfusion can be avoided if a lower threshold was used [10].

When assessing the use of restrictive transfusion practices among a gynecologic oncology patient population, investigations conducted by Boone et al. demonstrated that a restrictive transfusion protocol did not result in increased morbidity or mortality [11]. An additional study in 2015 analyzing National Surgical Quality Improvement Program data for all gynecologic cancer cases reported on composite morbidity and mortality and suggested an association between blood transfusions and increased surgical wound infections, wound disruption, pneumonia, unplanned intubation, pulmonary embolism, ventilator use greater than 48 hours, renal failure, urinary tract infections, deep vein thrombosis, sepsis, shock and mortality, favoring creation of institutional transfusion guidelines [12].

Quality improvement measures suggesting benefits of restrictive blood transfusion lead to the adaptation of standardized practices within our institution's gynecologic oncology department. A retrospective analysis was conducted pre- and post-adaptation to evaluate postoperative patient morbidity and mortality using National Surgical Quality Improvement Program data to establish the effect of these practices within this unique patient population.

\section{Methods}

The Gynecologic Oncology Restrictive Transfusion Protocol was implemented by our department based on multiple studies showing the preponderance of evidence highlighting the safety and feasibility of a restrictive transfusion protocol [10-12]. The Gynecologic Oncology Restrictive Transfusion Protocol required that no blood transfusions would be administered postoperatively for hemoglobin greater than $7.0 \mathrm{~g} / \mathrm{dL}$ (or hematocrit greater than $21.0 \%$ ) and that all packed red blood cells were administered in one unit increments followed by reevaluation of blood parameters [11]. Exceptions to this protocol included postoperative symptomatic anemia, active bleeding, postoperative severe sepsis, postoperative active coronary ischemia, and postoperative transfusion after 1.5 liter or greater blood loss [11]. Prior to implementation of the Gynecologic Oncology Restrictive Transfusion Protocol, all participating gynecologic oncology providers were educated on the rationale and intent of the protocol, as well as the medical literature supporting the safety and potential benefits of a restrictive practice.

Following approval by the Roswell Park Comprehensive Cancer Center Institutional Review Board a retrospective analysis of the National Surgical Quality Improvement Program database was performed evaluating all surgical patients treated on the gynecologic oncology service at our tertiary-care National Comprehensive Cancer Network designated Comprehensive Cancer Center from July $1^{\text {st }} 2011$ (the year National Surgical Quality Improvement Program reporting was introduced) until December $30^{\text {th }}$ 2016. Patients were categorized to "preprotocol" if they underwent surgery prior to January $1^{\text {st }} 2014$, or "post-protocol" after January $1^{\text {st }} 2014$ when the Gynecologic Oncology Restrictive Transfusion Protocol was implemented.
Hospital charge data related to blood transfusion were obtained through our institutional senior financial analysts.

The primary outcome of the study was the rate of packed red blood cell transfusions. Secondary outcomes included the National Surgical Quality Improvement Program reported variables which included superficial and deep surgical site infection, postoperative death, postoperative pneumonia, postoperative sepsis, postoperative urinary tract infections, unplanned intubation, venous thromboembolism, pulmonary embolism, stroke, cardiac arrest, acute renal failure, readmission rates and length of stay. Statistical analysis was performed in the R 3.4.1 statistical language. All statistical tests were twosided and used a nominal level of 0.05 for significance. $t$-tests were used to determine differences in continuous variables. Chi-squared tests or Fisher's exact tests were used to determine differences for categorical variables.

\section{Results}

Seven-hundred and fifty-five postoperative gynecologic patients in the National Surgical Quality Improvement Program database between 2011 and 2013 and 727 postoperative gynecologic patients between 2014 and 2016 were identified. The study population was well balanced with the exception of American Society of Anesthesiologists classification between groups, with the post-protocol group more likely to have American Society of Anesthesiologists 3-4 classification (Table 1). Mean preoperative hematocrit was lower in the post-protocol group at $38.7 \%$ compared to $39.2 \%$ in the pre-protocol group $(\mathrm{p}=0.03)$. Minimally invasive surgery surgical volume during study periods was not clinically significantly different with a $1.77 \%$ increase in minimally invasive cases in the two years following implementation of the gynecologic oncology restrictive transfusion protocol. Surgical faculty remained relatively constant over the study period with no faculty additions and one provider leaving the practice. The rate of blood transfusion (proportion of patients receiving one or more units of blood) in the pre-protocol group was $83 / 755(11.0 \%)$ compared to $43 / 427(5.9 \%)$ post-protocol implementation, reflecting a $48 \%$ reduction in use of postoperative blood transfusion $(\mathrm{p}<0.001)$.

A total of 244 units of packed red blood cells were given in the pre-protocol group over the study period versus 129 units given in the post-protocol group. At an estimated cost of $\$ 830$ per unit of packed red blood cells at our institution, the restrictive protocol accounted for an average cost savings of $\$ 17,620$ per year over our study period from decreasing blood transfusion alone. This is likely a large underestimation, as this table does not include indirect costs including skilled nursing assessment and monitoring around the time of transfusion, the cost of transfusion-associated medications such as diphenhydramine, acetaminophen, or furosemide, or any downstream costs that could be associated with longer hospital stay or readmission from related complications such as surgical site infections.

Post transfusion morbidity and mortality is illustrated in Table 2. Significant reductions were found in rates of both superficial and deep surgical site infection. The rate of superficial surgical site infection decreased from a rate of 7.68 per 100 surgical cases to 4.13 per 100 surgical cases after implementation of the Gynecologic Oncology Restrictive Transfusion Protocol, a 


\begin{tabular}{|c|c|c|c|}
\hline & $\begin{array}{l}\text { Pre-Protocol } \\
(\mathrm{N}=755)\end{array}$ & $\begin{array}{l}\text { Post-Protocol } \\
(\mathrm{N}=727)\end{array}$ & p-value \\
\hline Mean Age & 56.1 & 57.3 & 0.09 \\
\hline Race & & & 0.06 \\
\hline White & 649 & 607 & \\
\hline African American & 64 & 65 & \\
\hline Asian & 2 & 9 & \\
\hline American Indian & 5 & 13 & \\
\hline Other/Unknown & 35 & 33 & \\
\hline Mean Body Mass Index & 32.9 & 32.4 & 0.27 \\
\hline ASA Classification & & & 0.003 \\
\hline 1 & 16 & 22 & \\
\hline 2 & 539 & 453 & \\
\hline 3 & 194 & 244 & \\
\hline 4 & 6 & 8 & \\
\hline $\begin{array}{l}\text { Mean Preoperative } \\
\text { Hematocrit }\end{array}$ & 39.2 & 38.7 & 0.03 \\
\hline Smoking Status & & & 0.33 \\
\hline Yes & 163 & 141 & \\
\hline No & 592 & 586 & \\
\hline $\begin{array}{c}\text { History of Diabetes } \\
\text { Mellitus }\end{array}$ & & & 0.82 \\
\hline Yes & 109 & 101 & \\
\hline No & 646 & 626 & \\
\hline Functional Status & & & 0.45 \\
\hline Independent & 744 & 722 & \\
\hline Partially Dependent & 9 & 4 & \\
\hline Totally Dependent & 2 & 1 & \\
\hline
\end{tabular}

Table 1: Implementation of a restrictive blood transfusion protocol in a gynecologic oncology service: Patient demographics.

$48 \%$ reduction between the pre-protocol and protocol groups $(\mathrm{p}=0.005)$. Deep surgical site infections similarly decreased by $70.6 \%$ between pre-and post-protocol groups at a rate of 2.25 per 100 surgical cases to 0.69 per 100 surgical cases respectively $(\mathrm{p}=0.02)$. Median length of hospitalization decreased by $33 \%$ (3.0 days $v s 2.0$ days pre-protocol and post-protocol, $\mathrm{p}<0.001$ ).

Reductions in rates of postoperative pneumonia $(2.0 \% v s$ $1.1 \%)$, sepsis $(1.6 \%$ vs $1.0 \%)$, unplanned intubation $(0.9 \%$ vs $0.6 \%)$, pulmonary embolism $(1.2 \%$ vs $1.1 \%)$, progressive renal insufficiency $(0.3 \%$ vs $0.1 \%)$, urinary tract infections $(3.0 \%$ vs $0.1 \%)$, stroke $(0.1 \%$ vs $0 \%)$, cardiac complications $(0.4 \%$ vs $0.1 \%)$, venous thromboembolism ( $1.2 \%$ vs $1.0 \%)$, death within 30 days of surgery $(0.8 \%$ vs $0.4 \%)$ and readmission rates $(7.5 \%$ vs $6.1 \%$ ) in the post-protocol group were also observed; however these findings did not reach statistical significance (Table 2).

\section{Discussion}

The implementation of a restrictive transfusion policy in an academic gynecologic oncology practice is safe and feasible. Limiting transfusion to patients with a hemoglobin value less than $7 \mathrm{~g} / \mathrm{dL}$ with unit-by-unit reassessment of blood parameters resulted in a significant reduction in blood product transfusion for a gynecologic service with marked reduction in surgical site infection rates and length of hospitalization. An increase in death within 30 days of surgery, cardiovascular complications, or infections was not observed, in contrast to previous studies among surgical oncology patients [13].

Ultimately, reducing patient morbidity should be the goal of all institutions and any cost savings related to this protocol are second to improving patient outcomes. From an institutional financial standpoint blood transfusions are expensive, and reducing blood transfusion provides an opportunity to reduce both direct and indirect costs of transfusion and associated patient complications ranging from surgical site infection to perioperative death. Direct transfusion-associated costs were estimated at a minimum of $\$ 17,620$ per year in savings over the study period. The true savings is likely many times more as this number does not account for the indirect costs of blood transfusions related to overhead and any patient complications. Adding the average per day hospital charge for a non-intensive care unit bed or step-down unit the reduction in hospital stay amounted to at least an average savings of $\$ 90,037$ per year.

A significant improvement in patient outcome variables such as surgical site infection (superficial and deep) was additionally observed, which was also associated with healthcare cost savings. One study highlighted that one superficial surgical site infection costs over $\$ 7,000$ and one deep surgical site infection can cost over $\$ 25,000$ [14]. Using these associated costs the combined potential savings relating to surgical site infection reductions was an average of $\$ 91,513$ per year over our study period. It is acknowledged that correlation does not imply causation and there are other possible explanations for a decrease in some of these outcomes. It would be impossible to isolate and control for these variables in a real-world scenario reported here. Importantly, other restrictive transfusion studies have observed similar surgical site infection improvements $[11,12]$. There could be a biologic explanation for this reduction in wound infections. Transfusion-related immunomodulation has been described after allogeneic blood transfusions and is associated with impaired natural killer cell function, decreased phagocytosis by macrophages, suppression of lymphocyte production and antigen presentation, all of which can impair wound healing. Therefore fewer blood transfusions may result in less immunosuppression which could contribute to lower surgical site infection rates [15].

A weakness of this study is that it was retrospective and lacked the strength of prospectively randomized control trials. Nevertheless these findings are similar to the conclusions of mixed population studies [10-12]. Although reductions in postoperative morbidity are clearly demonstrated, there may be multiple factors other than decreased blood transfusion that could account for these improvements. For example, a surgical site infection task force was introduced during the study period which introduced multiple interventions (including patient education, preoperative body wash, a negative pressure wound dressing for obese patients, and a special closure tray).

Additionally, high-level evidence has shown that enhanced recovery pathways and enhanced recovery after surgery protocols can decrease postoperative morbidity and length of stay [16]. An enhanced recovery after surgery protocol was implemented in January of 2016 which coincided with the current study period, making this a possible contributor to the favorable morbidity observed. All significant reductions in morbidity were related to surgical site infections, both superficial and deep. Although significance reductions in other measures of postoperative morbidity (see results) were not observed, these may be demonstrated with a larger sample size.

Evolving surgical techniques are another possible explanation accounting for improved morbidity during the study period. 
Citation: Mark J, Lynam S, Morrell K, et al. Implementation of a restrictive blood transfusion protocol in a gynecologic oncology service.. Gynecol Reproduct Endocrinol -UK. 2019;3(1):1-5

\begin{tabular}{|c|c|c|c|}
\hline & Pre-Protocol & Post-Protocol & p-value \\
\hline Transfused with pRBC & & & $<0.001$ \\
\hline Yes & 83 & 43 & \\
\hline No & 672 & 684 & \\
\hline Mean pRBC Transfused & 2.9 & 3.0 & 0.90 \\
\hline Superficial Surgical Site Infection & & & 0.005 \\
\hline Yes & 58 & 30 & \\
\hline No & 697 & 697 & \\
\hline Deep Surgical Site Infection & & & 0.02 \\
\hline Yes & 17 & 5 & \\
\hline No & 738 & 722 & \\
\hline Post-Op Pneumonia & & & 0.24 \\
\hline Yes & 15 & 8 & \\
\hline No & 740 & 719 & \\
\hline Post-Op Sepsis & & & 0.40 \\
\hline Yes & 12 & 7 & \\
\hline No & 743 & 720 & \\
\hline Post-Op Unplanned Intubation & & & 0.59 \\
\hline Yes & 7 & 4 & \\
\hline No & 748 & 723 & \\
\hline Post-Op Pulmonary Embolism & & & 1.00 \\
\hline Yes & 9 & 8 & \\
\hline No & 746 & 719 & \\
\hline Post-Op $>48 \mathrm{~h}$ on Ventilator & & & 0.50 \\
\hline Yes & 3 & 5 & \\
\hline No & 752 & 722 & \\
\hline Post-Op Progressive Renal Insufficiency & & & 1.00 \\
\hline Yes & 2 & 1 & \\
\hline No & 753 & 726 & \\
\hline Post-Op Acute Renal Failure & & & 0.49 \\
\hline Yes & 0 & 1 & \\
\hline No & 755 & 726 & \\
\hline Post-Op Urinary Tract Infection & & & 0.30 \\
\hline Yes & 23 & 15 & \\
\hline No & 732 & 712 & \\
\hline Post-Op Cerebral Vascular Accident & & & 1.00 \\
\hline Yes & 1 & 0 & \\
\hline No & 754 & 727 & \\
\hline Post-Op Cardiac Arrest & & & 1.00 \\
\hline Yes & 2 & 1 & \\
\hline No & 753 & 726 & \\
\hline Post-Op Myocardial Infarction & & & 0.49 \\
\hline Yes & 0 & 1 & \\
\hline No & 755 & 726 & \\
\hline Post-Op Venous Thromboembolism & & & 0.86 \\
\hline Yes & 9 & 7 & \\
\hline No & 746 & 720 & \\
\hline Post-Op Death within $30 \mathrm{~d}$ & & & 0.51 \\
\hline Yes & 6 & 3 & \\
\hline No & 749 & 724 & \\
\hline Median Length of Stay (days) & 3.0 & 2.0 & $<0.001$ \\
\hline Readmission Within $30 \mathrm{~d}$ of Surgery & & & 0.30 \\
\hline Yes & 57 & 44 & \\
\hline No & 698 & 683 & \\
\hline
\end{tabular}

Table 2: Postoperative blood transfusion metrics and outcomes.

For example, it is well established that minimally invasive surgery compared to laparotomy results in shorter hospital stays, reductions in postoperative morbidity as well as blood transfusion rates according to many studies [17-20]. However, there were no changes in surgical faculty during the study period and minimally invasive surgical volume did not markedly change. Therefore, surgical approach is less likely to play a significant role in the favorable morbidity observed in this 
population.

Another potential weakness of this study is that the gynecologic oncology restrictive transfusion protocol compliance rates could not be accurately tracked due to the use of National Surgical Quality Improvement Program data. However, reductions in relevant postoperative morbidity between the two study groups favoring the patients in the Gynecologic Oncology Restrictive Transfusion Protocol was demonstrated, which is consistent with high protocol compliance. It must also be acknowledged that $58 / 755(7.7 \%)$ of patients in the pre-protocol arm and $62 / 727(8.5 \%)$ in the post-protocol arm did not have preoperative hematocrits available in the database; therefore the average preoperative hematocrits might not reflect the true average. An additional weakness of our study was not being able to demonstrate significant reductions in all of the secondary outcomes which we attribute to our study not being powered sufficiently to detect these differences.

Strengths of this study include the large patient numbers, controlled surgeons, and use of National Surgical Quality Improvement Program data which ensure the accuracy of findings since data capture and reporting is standardized. The inclusion of cost information surrounding the savings associated with the gynecologic oncology restrictive transfusion protocol highlights the potential impact if restrictive protocols are implemented on a larger scale. Another unique aspect of this study is that it focused solely on surgical patients, which would make these findings applicable and reproducible to this patient population. Additional trials could be conducted to specifically evaluate gynecologic oncology medical patients to discern if the morbidity reductions are similar.

Given the encouraging results without any increase in patient morbidity or mortality using the gynecologic oncology restrictive transfusion protocol, future directions include the expansion of this conservative transfusion protocol hospitalwide and potentially even city-wide. In the spirit of all quality improvement endeavors, ongoing evaluation of patient outcomes and provider compliance through query of patient hospital records will continue to ensure positive findings and potentially identify areas for improvement.

\section{Acknowledgement}

This work was supported by Roswell Park Comprehensive Cancer Center with the National Cancer Institute grant P30CA016056 and the Roswell Park Alliance Foundation and National Cancer Institute training grant T32CA108456 in surgical oncology

\section{References}

1. Scully RE, Schoenfeld AJ, Jiang W, et al. Defining optimal length of opioid pain medication prescription after common surgical procedures. JAMA Surg. 2018;153:37-43.

2. MD RG. Clinical practice guidelines from the AABB: Red blood cell transfusion thresholds and storage. Blood News. 2018.

3. Kowalczyk WJ, Sullivan MA, Evans SM, et al. Sex differences and hormonal influences on response to mechanical pressure pain in humans. J Pain. 2010;11:330-42.

4. Carson JL, Guyatt G, Heddle NM, et al. Clinical practice guidelines from the AABB: Red blood cell transfusion thresholds and storage.
JAMA. 2016;316:2025-35.

5. Updates on blood transfusion guidelines. Lancet Haematol. 2016;3:e547.

6. Carson JL, Willett LR. Is a hemoglobin of $10 \mathrm{~g} / \mathrm{dL}$ required for surgery? Med Clin North Am. 1993;77:335-47.

7. Sahu S, Hemlata, Verma A. Adverse events related to blood transfusion. Indian J Anaesth. 2014;58:543-51.

8. Vamvakas EC, Blajchman MA. Transfusion-related mortality: The ongoing risks of allogeneic blood transfusion and the available strategies for their prevention. Blood. 2009;113:3406-17.

9. Hebert PC, Wells G, Blajchman MA, et al. A multicenter, randomized, controlled clinical trial of transfusion requirements in critical care. Transfusion requirements in critical care investigators, Canadian critical care trials group. N Engl J Med. 1999;340:409-17.

10.Carson JL, Stanworth SJ, Roubinian N, et al. Transfusion thresholds and other strategies for guiding allogeneic red blood cell transfusion. Cochrane Database Syst Rev. 2016;10:CD002042.

11.Boone JD, Kim KH, Marques M, et al. Compliance rates and outcomes associated with a restrictive transfusion policy in gynecologic oncology patients. Gynecol Oncol. 2014;132:227-30.

12.Prescott LS, Aloia TA, Brown AJ, et al. Perioperative blood transfusion in gynecologic oncology surgery: Analysis of the national surgical quality improvement program database. Gynecol Oncol. 2015;136:65-70.

13.de Almeida JP, Vincent JL, Galas FR, et al. Transfusion requirements in surgical oncology patients: A prospective, randomized controlled trial. Anesthesiol. 2015;122:29-38.

14.Schweizer ML, Cullen JJ, Perencevich EN, et al. Costs associated with surgical site infections in veterans affairs hospitals. JAMA Surg. 2014;149:575-81.

15.Hellings S, Blajchman MA. Transfusion-related immunosuppression. Anaesthesia Intens Care Med. 2009;10:231-34.

16.Barber EL, Van Le L. Enhanced recovery pathways in gynecology and gynecologic oncology. Obstet Gynecol Surv. 2015;70:780-92.

17.Zullo F, Falbo A, Palomba S. Safety of laparoscopy vs laparotomy in the surgical staging of endometrial cancer: A systematic review and meta-analysis of randomized controlled trials. Am J Obstet Gynecol. 2012;207:94-100.

18.Mori KM, Neubauer NL. Minimally invasive surgery in gynecologic oncology. ISRN Obstet Gynecol. 2013;2013:312982.

19.Diver E, Hinchcliff E, Gockley A, et al. Minimally invasive radical hysterectomy for cervical cancer is associated with reduced morbidity and similar survival outcomes compared with laparotomy. J Minim Invasive Gynecol. 2017;24:402-6.

20.Sinno AK, Fader AN. Robotic-assisted surgery in gynecologic oncology. Fertil Steril. 2014;102:922-32.

\section{*Correspondence to:}

Jaron Mark

Roswell Park Comprehensive Cancer Center

Department of Gynecologic Oncology

Buffalo, New York

USA

Tel: 7168453497

E-mail: jaron.mark@roswellpark.org 